\title{
A Multiband Diversity Antenna System for Compact Mobile/Wireless Devices: Modeling and Performance Evaluation
}

\author{
M. Karaboikis, V. Papamichael, C. Soras, and V. Makios \\ Laboratory of Electromagnetics, Department of Electrical and Computer Engineering, University of Patras, 26500 Patras, Greece
}

Correspondence should be addressed to C. Soras, soras@ece.upatras.gr

Received 1 August 2007; Accepted 3 December 2007

Recommended by Jaume Anguera

A novel compact multiband diversity antenna system appropriate for integration onto space-limited devices is presented. It comprises three pairs of printed fractal Minkowski and Koch monopole antennas which resonate in the 1.67-2, 2.3-2.56 and 4.55$5.55 \mathrm{GHz}$ bands, respectively, supporting thus a large variety of wireless technologies for mobile and wireless local area networks (WLANs) applications. The operational characteristics of the antenna system were extracted by simulation using a method-ofmoment- (MoM-) based electromagnetic field solver. The diversity performance in a uniform multipath environment under the maximal-ratio combining (MRC) scheme is also evaluated for the two extreme modes of operation, that is, when all three antenna pairs operate simultaneously (worst case) and when only one pair is active while the others being open-circuited (best case). The calculated effective diversity gain (EDG) for the worst/best case is 5.8/6.4, 5.8/7.2 and 7.1/7.5 dB for the 1.8, 2.4 and 5.2 GHz frequencies, respectively.

Copyright (C) 2008 M. Karaboikis et al. This is an open access article distributed under the Creative Commons Attribution License, which permits unrestricted use, distribution, and reproduction in any medium, provided the original work is properly cited.

\section{INTRODUCTION}

Multiradio antenna systems which support multiple wireless standards are the trend for future communication systems. The performance of these systems can be significantly enhanced using antenna diversity, which combats efficiently the multipath fading. Dual-band diversity antennas which operate in 2.4 and $5.2 \mathrm{GHz}$ industrial, scientific, and medical (ISM) frequency bands have been extensively reported in literature (e.g., [1-4]). The attention to multiband diversity antenna systems, on the other hand, is currently very limited $[5,6]$.

In [5], two planar inverted-F antenna (IFA) arrays, which cover the 2.4 and 5.25/5.8 GHz ISM bands, are proposed for WLAN applications. In [6], the proposed multiband diversity antenna is very compact and covers in addition the $1.8 \mathrm{GHz}$ band, which supports digital wireless protocols and thus can be used for mobile applications as well. However, in both $[5,6]$ the performance evaluation of the investigated multiband diversity antenna systems, when operating in multipath fading environments, is not presented.
In this study, a novel compact multiband diversity antenna system suitable for integration on space-limited mobile/wireless devices is presented along with its diversity performance evaluation. The antenna system is formulated using printed Koch and Minkowski fractal monopoles which were selected due to their extremely compact dimensions [79] and can be integrated onto a device almost at no cost. The modeling of the proposed antenna structure was performed using a method-of-moment- (MoM-) based commercial electromagnetic software [10]. This multielement antenna (MEA) system covers a wide range of frequencies (1.67-2, 2.3-2.56, and 4.55-5.55 GHz) which can support the following wireless technologies: GSM and DCS (1.67$2 \mathrm{GHz}), 802.11 \mathrm{~b} / \mathrm{g} / \mathrm{n}$, Wi-Fi and Bluetooth $(2.3-2.55 \mathrm{GHz})$, and $802.11 \mathrm{a} / \mathrm{j}$ and HIPERLAN/2 (4.55-5.55 GHz) [11, Chapter 2]. During its operation in these bands, two extreme modes are investigated in detail: in the first mode, the antennas operate simultaneously in the three bands (worst case); while in the second mode only one band is active (best case).

The paper is organized as follows. In Section 2, the methodology for evaluating the diversity performance under 
the maximal-ratio combining (MRC) scheme is described. Specifically, the effective diversity gain (EDG) [12] is evaluated through the signals covariance matrix, which is formulated by means of the complex correlation coefficient $\left(\rho_{c}\right)$ of the received signals [13] and the mean effective gain (MEG) [14] of the antenna elements. Section 3 presents the design of the proposed antenna system along with its operational characteristics. Section 4 presents the simulated diversity performance in a uniform propagation environment for the two extreme modes of operation. The study is summarized in Section 5.

\section{TWO-BRANCH MAXIMAL-RATIO COMBINING DIVERSITY PERFORMANCE EVALUATION METHODOLOGY}

In this section, the metrics for the diversity performance evaluation are presented. These metrics are the envelope correlation coefficient $\left(\rho_{e}\right)$ of the received signals, the mean effective gain(MEG) of the antenna elements, and the achieved effective diversity gain (EDG) under the MRC scheme, which as well known provides the upper bound on diversity performance.

The envelope correlation coefficient between the two antenna branches can be calculated through the complex correlation coefficient by [13].

$$
\begin{aligned}
\rho_{e 12} & \approx\left|\rho_{c 12}\right|^{2}, \\
\rho_{c 12} & =\frac{\oiiint Z(\Omega) d \Omega}{\sqrt{\oiiint Q_{1}(\Omega) d \Omega \oiiint Q_{2}(\Omega) d \Omega}}, \\
Z(\Omega) & =\Gamma \cdot E_{\theta 1}(\Omega) \cdot E_{\theta 2}^{*}(\Omega) \cdot P_{\theta}(\Omega)+E_{\varphi 1}(\Omega) \cdot E_{\varphi 2}^{*}(\Omega) \cdot P_{\varphi}(\Omega), \\
Q_{1}(\Omega) & =\Gamma \cdot G_{\theta 1}(\Omega) \cdot P_{\theta}(\Omega)+G_{\varphi 1}(\Omega) \cdot P_{\varphi}(\Omega), \\
Q_{2}(\Omega) & =\Gamma \cdot G_{\theta 2}(\Omega) \cdot P_{\theta}(\Omega)+G_{\varphi 2}(\Omega) \cdot P_{\varphi}(\Omega),
\end{aligned}
$$

where $\mathrm{E}_{\theta}$ and $\mathrm{E}_{\varphi}$ are the $\theta$ and $\varphi$ polarized, realized (i.e., mismatch included), active [15] (i.e., mutual coupling included) electric field patterns of the antennas; $\Gamma$ is the crosspolarization power ratio (XPR) ofthe incident waves; $P_{\theta}$ and $P_{\varphi}$ are the $\theta$ and $\varphi$ components of the angular density functions of the incoming plane waves; $G_{\theta}$ and $G_{\varphi}$ are the $\theta$ and $\varphi$ polarized components of the antennas realized active power gain patterns; and $\Omega$ is the solid angle. The asterisk denotes the complex conjugate.

The MEG of an antenna element, which is defined as the ratio of the mean received $\left(P_{\text {rec }}\right)$ to the mean incident power at element $\left(P_{\text {inc }}\right)$, can be calculated from [14]

$$
\begin{aligned}
\operatorname{MEG}_{1,2} & =\frac{P_{\text {recl,2 }}}{P_{\text {inc }}} \\
& =\oint\left[\frac{\Gamma}{1+\Gamma} P_{\theta}(\Omega) G_{\theta 1,2}(\Omega)+\frac{1}{1+\Gamma} P_{\varphi}(\Omega) G_{\varphi 1,2}(\Omega)\right] d \Omega .
\end{aligned}
$$

In the case of a uniform propagation environment where $\Gamma=$ 1 and $P_{\theta}=P_{\varphi}=1 / 4 \pi$, (2) simplifies to

$$
\mathrm{MEG}_{1,2}=\frac{e_{\mathrm{tot} 1,2}}{2},
$$

with $e_{\text {tot } 1,2}$ being the total efficiency of the respective antenna element. In turn, $e_{\text {tot } 1,2}$ is defined as the product of the antenna mismatch efficiency; $e_{\text {mis } 1,2}$ times the radiation efficiency $e_{\mathrm{rad} 1,2}$ (which incorporates the conduction and dielectric losses):

$$
e_{\mathrm{tot} 1,2}=e_{\mathrm{mis} 1,2} \cdot e_{\mathrm{rad} 1,2}
$$

Diversity performance, which is the ability of a multielement antenna system to mitigate fading, can be achieved when the following two criteria are met [16]:

$$
\rho_{e 12}<0.5, \quad \frac{\mathrm{MEG}_{1}}{\mathrm{MEG}_{2}} \cong 1 .
$$

A widely used measure of the diversity performance of different antenna systems is the effective diversity gain (EDG) which is defined mathematically as [12].

$$
\mathrm{EDG}=\left.\frac{P_{\text {div }}}{P_{\text {ideal }}}\right|_{p \%},
$$

where $P_{\text {div }}$ is the received power of the combined signal, and $P_{\text {ideal }}$ is the power received by an ideal antenna operating in the same environment. In this paper, the ideal antenna is a dual-polarized isotropic radiator with unit radiation efficiency [14]. $P_{\text {div }}$ and $P_{\text {ideal }}$ are calculated at the same probability level $(p \%)$ in a cumulative probability density versus relative power-level plot. The cumulative density function (CDF) of $P_{\text {ideal }}$ follows the Rayleigh distribution while the CDF of $P_{\text {div }}$ follows the distribution of MRC signals which is given by [17, pages 364-366], [18] as follows:

$$
P(\gamma \leq x)=\frac{1}{\lambda_{1}-\lambda_{2}}\left[\lambda_{1}\left\{1-e^{-x / \lambda_{1}}\right\}-\lambda_{2}\left\{1-e^{-x / \lambda_{2}}\right\}\right] .
$$

In (7), $\lambda_{1,2}$ are the eigenvalues of the signals covariance matrix $\Lambda$ which is formulated using the calculated values $\rho_{c}$ of and MEGs,

$$
\boldsymbol{\Lambda}=P_{\mathrm{inc}}\left[\begin{array}{cc}
\mathrm{MEG}_{1} & \rho_{c 12} \sqrt{\mathrm{MEG}_{1} \mathrm{MEG}_{2}} \\
\rho_{c 12}^{*} \sqrt{\mathrm{MEG}_{1} \mathrm{MEG}_{2}} & \mathrm{MEG}_{2}
\end{array}\right] .
$$

For the M-branch MRC case, the covariance matrix-based methodology for evaluating EDG can be found in $[19,20]$.

\section{COMPACT PRINTED MULTIELEMENT ANTENNA SYSTEM DESIGN}

The geometry and dimensions of the investigated configuration are depicted in Figure 1. It has the typical dimensions of a PC card, whereas the dimensions of the ground plane are $46 \mathrm{~mm} \times 89 \mathrm{~mm}$. The system consists of two $35 \mu \mathrm{m}$ thick copper layers with the antennas placed at the upper one and the ground plane at the bottom. The antennas are printed at 


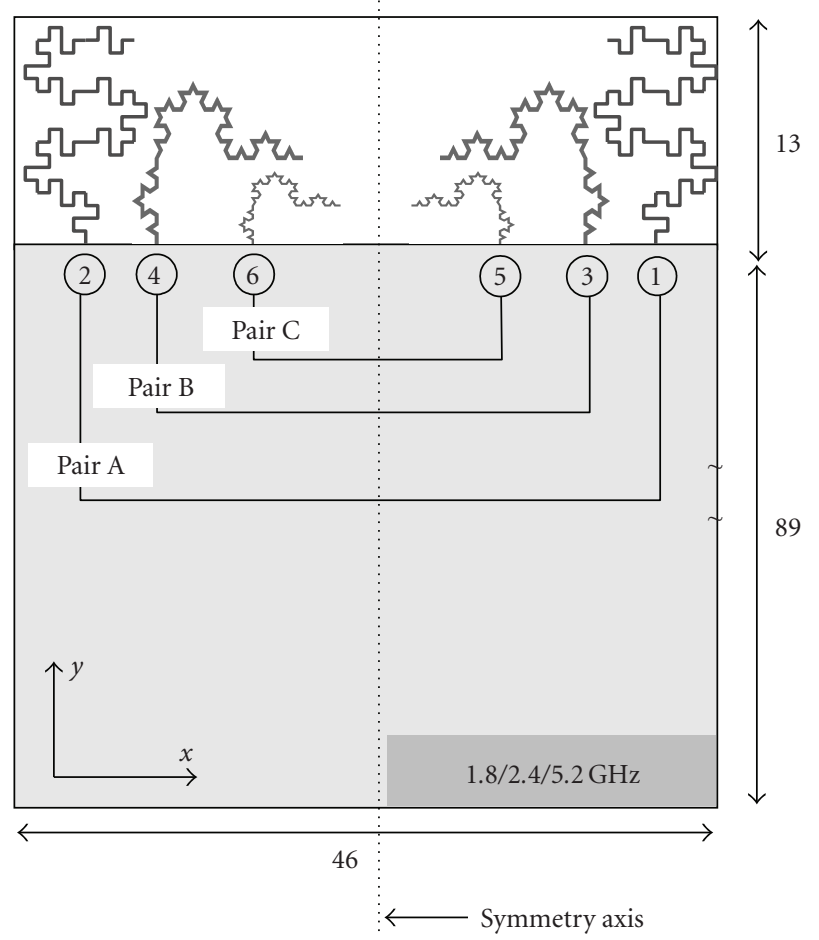

(a)
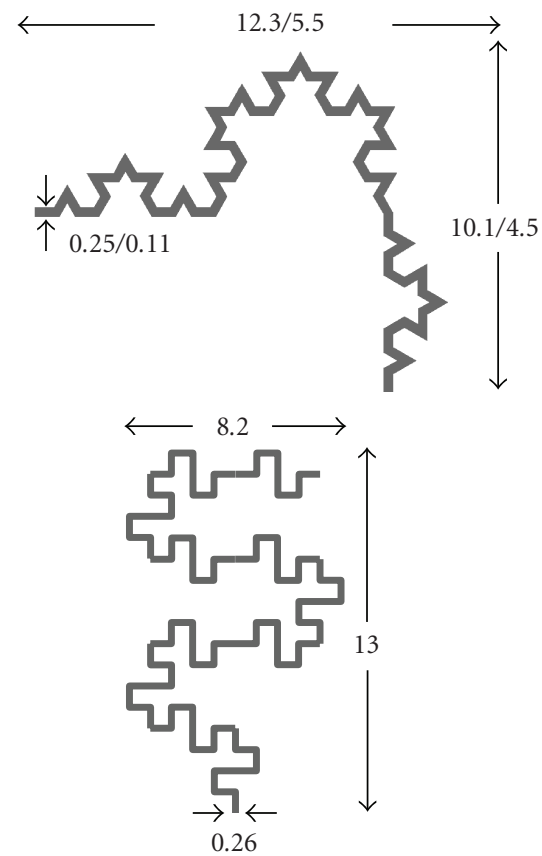

Dimensions ( $\mathrm{mm})$

(b)

FIGURE 1: Geometry and dimensions of the multiband antenna diversity system.

the edge of the device's ground plane on an 8-mil-thick substrate with $\varepsilon_{r}=3.38$ and $\tan \delta=0.0027$.

The antennas used are the folded Koch monopole [7] and the modified Minkowski monopole of the second iteration
$[8,9]$. Both monopoles were chosen primarily due to their extremely compact size (Minkowski: $\left(\lambda_{0} / 10\right) \times\left(\lambda_{0} / 18\right)$; and Koch: $\left.\left(\lambda_{0} / 10\right) \times\left(\lambda_{0} / 12\right)\right)$, given the restricted space available on the mobile/wireless terminal devices. These fractal antennas provide in addition wide bandwidth, omnidirectional radiation patterns, and ease of fabrication with no additional cost.

The configuration depicted in Figure 1 was simulated using IE3D, a commercial method-of-moment-based electromagnetic field solver [10]. The pairs A, B, and C are well tuned at the $1.67-2 \mathrm{GHz}, 2.3-2.56 \mathrm{GHz}$, and $4.55-5.55 \mathrm{GHz}$ frequency bands, respectively, as derived from the computed $S_{\text {ii }}$ parameters of Figure 2. Apart from the $S_{\text {ii }}$ parameters, the mutual coupling ( $S_{i j}$ parameters) between the elements comprising each antenna pair (interpair coupling) is also depicted in Figure 2. It can be observed that despite the fact that the physical distance between the antenna elements from pair A to C decreases, the interpair coupling also decreases. This is attributed to the increase of the electrical distance between the elements of the antenna pairs as the frequency increases. Table 1 depicts the radiation efficiency of the antenna elements proving that these antennas are excellent radiators despite their miniature size. Due to the symmetrical placement of the antennas, the presented results are valid for both elements that comprise each antenna pair.

Aside from the interpair coupling, the mutual coupling between neighboring elements which resonate in different frequency bands, hereafter called interband coupling, is also present and affects the antennas operation. As depicted in Figure 3 , in the frequencies where neither of any two antenna pairs resonate, the interband coupling among them is negligible3. Pairs $\mathrm{A}$ and $\mathrm{C}$ exhibit also negligible interband coupling at both 1.8 and $5.2 \mathrm{GHz}$ frequencies due to the fact that their "frequency distance" is the largest $(3.4 \mathrm{GHz})$. The increased interband coupling between pairs $\mathrm{A}$ and $\mathrm{C}$ for frequencies below $5 \mathrm{GHz}$ is attributed to a higher-order resonance of pair A that occurs at $4.5 \mathrm{GHz}$. From Figures 3(a) and 3(b), it can be concluded that pairs A and B exhibit the highest values $(\sim-8 \mathrm{~dB})$ of interband coupling at 1.8 and $2.4 \mathrm{GHz}$ which is caused by their relatively small "frequency distance" $(\sim 600 \mathrm{MHz})$. The interband coupling between pairs $\mathrm{C}$ and $\mathrm{B}$ is also negligible at both 2.4 and $5.2 \mathrm{GHz}$ due to their large "frequency distance" $(2.8 \mathrm{GHz})$.

\section{DIVERSITY PERFORMANCE IN A UNIFORM ENVIRONMENT}

In this section, the performance of the configuration presented in Figure 1 when operating in a receive diversity system is evaluated for two extreme operation modes. In the first mode, the device is assumed to operate simultaneously in three representative frequencies $(1.8,2.4$, and $5.2 \mathrm{GHz})$, and thus all antenna elements are terminated to $50 \Omega$ loads. In the second mode of operation, on the other hand, only one pair is active whiles the remaining two antenna pairs are open-circuited. The first mode is characterized as the worst case of operation, since a great amount of power is lost on the termination loads of the antenna pairs that operate at the other frequencies, whereas the second one as the best case via 


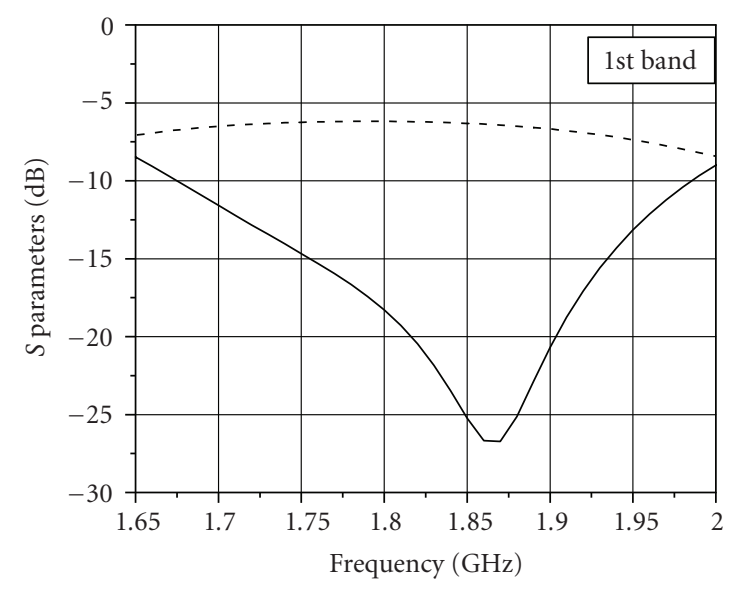

$-S_{11}$

(a)

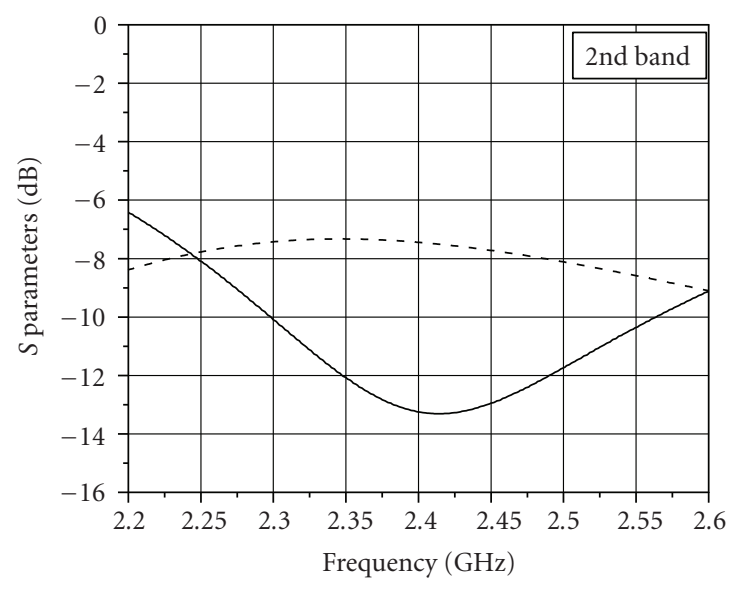

$-s_{33}$

(b)

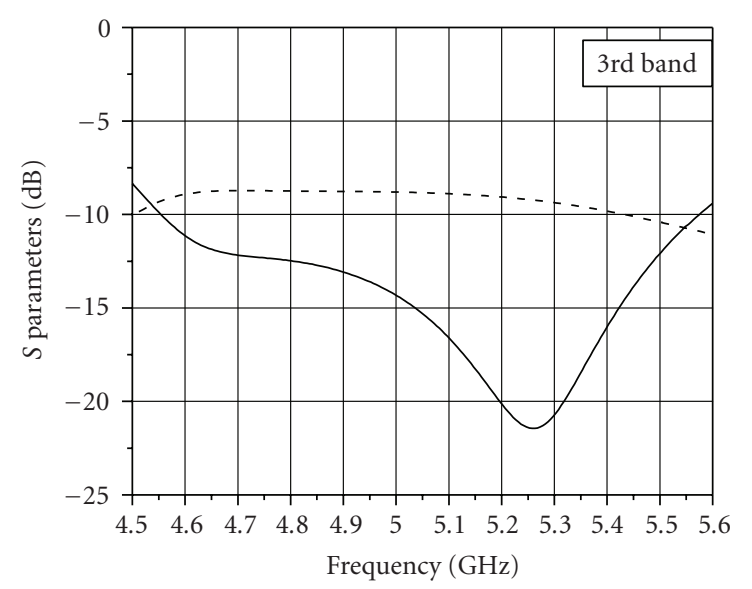

$-\mathrm{S}_{55}$

$-S_{56}$

(c)

FIGURE 2: Simulated $S_{\text {ii }}$ and $S_{\text {ij }}$ (interpair coupling) parameters of the three antenna pairs.

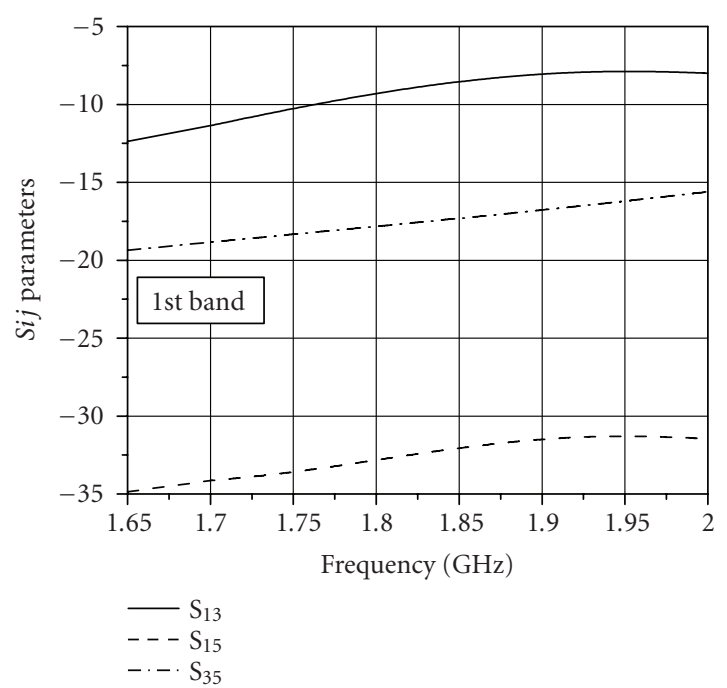

(a)

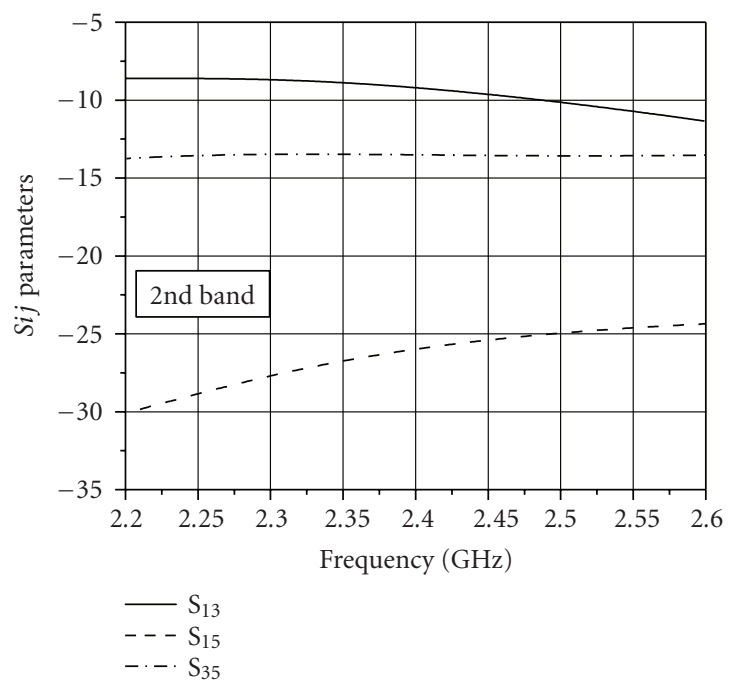

(b)

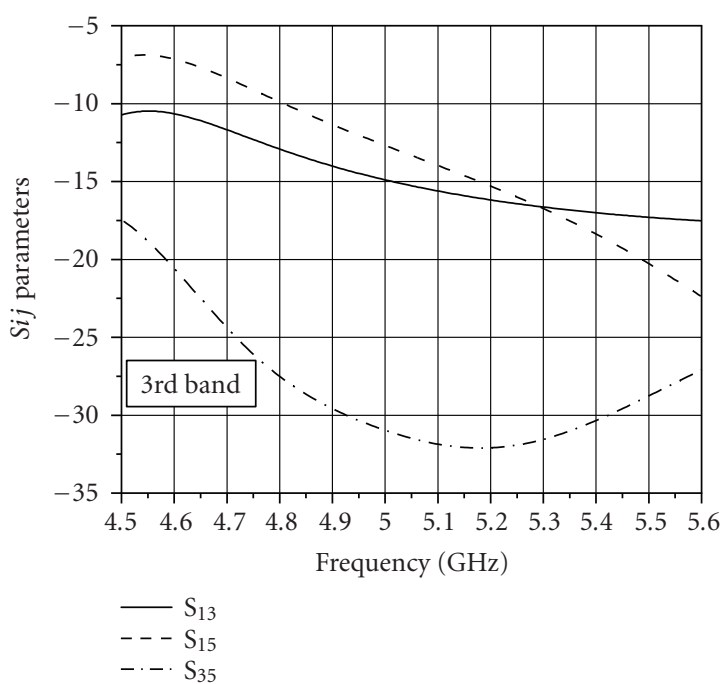

(c)

FIGURE 3: Simulated $S_{\text {ij }}$ parameters (interband coupling) between any two antenna pairs in the three frequency bands. 
TABLE 1: Radiation efficiency of the multiband diversity system's antennas.

\begin{tabular}{lcc}
\hline Frequency $(\mathrm{GHz})$ & Pair & $e_{\mathrm{rad}}(\%)$ \\
\hline 1.8 & A & 91.7 \\
2.4 & B & 91.4 \\
5.2 & C & 91.5 \\
\hline
\end{tabular}

TABle 2: Total efficiency, correlation coefficient, mean effective gains, and effective diversity gain of the multiband diversity system for the worst case.

\begin{tabular}{lccccc}
\hline Pair & Frequency $(\mathrm{GHz})$ & $e_{\mathrm{tot}}(\%)$ & $\rho_{e}$ & MEG & EDG $(\mathrm{dB})$ \\
\hline A & 1.8 & 52 & 0.042 & -5.6 & 5.8 \\
B & 2.4 & 52 & $2.2 \times 10^{-3}$ & -5.6 & 5.8 \\
C & 5.2 & 73 & 0.012 & -4.4 & 7.1 \\
\hline
\end{tabular}

the reverse reasoning. The MRC performance is investigated using the metrics presented in Section 2 in a uniform propagation environment, which has been proved to be a fair approximation for many indoor and outdoor propagation scenarios [20].

\subsection{Worst case of operation}

In this mode of operation, the three antenna pairs operate simultaneously being all terminated to $50 \Omega$ loads. The simulated realized active power gain patterns of the antenna elements on the azimuth $(\mathrm{x}-\mathrm{y})$ plane are shown in Figure 4. The complementary performance of the antenna pairs due to symmetry of the configuration is obvious, proving that it is a hybrid space-pattern diversity system with omnidirectional antennas. The total efficiencies of the elements, the envelope correlation coefficients, as well as the mean effective gains of the antennas are shown in Table 2. The MEG ratio in every case is unity indicating that the mean power received by each antenna branch is the same. This is attributed to the fact that the elements of the antenna pairs have symmetrical radiation patterns and operate in a uniform propagation environment. The calculated values of $\rho_{e}$ indicate that all antenna branches receive uncorrelated signals since $\rho_{e}$ is far below 0.5 , which is attributed to the inherent space-pattern diversity (see Figure 4).

Regarding the effective diversity gain, the antenna pair $\mathrm{C}$ exhibits the best performance with $7.1 \mathrm{~dB}$ of effective diversity gain at $5.2 \mathrm{GHz}$, whereas pairs $A$ and $B$ provide $5.8 \mathrm{~dB}$ gains at 1.8 and $2.4 \mathrm{GHz}$, respectively, (see Table 2). This gain reduction is attributed solely to the additive impact of the interpair and interband mutual couplings, which lowers the total efficiencies of these pairs and thus their MEG values as in (3). For pair C, however, both interpair and interband couplings have less impact as presented in Section 3 which is reflected to the higher EDG value.

\subsection{Best case of operation}

In this mode of operation, only one antenna pair operates with its elements being terminated to $50 \Omega$ loads whiles the

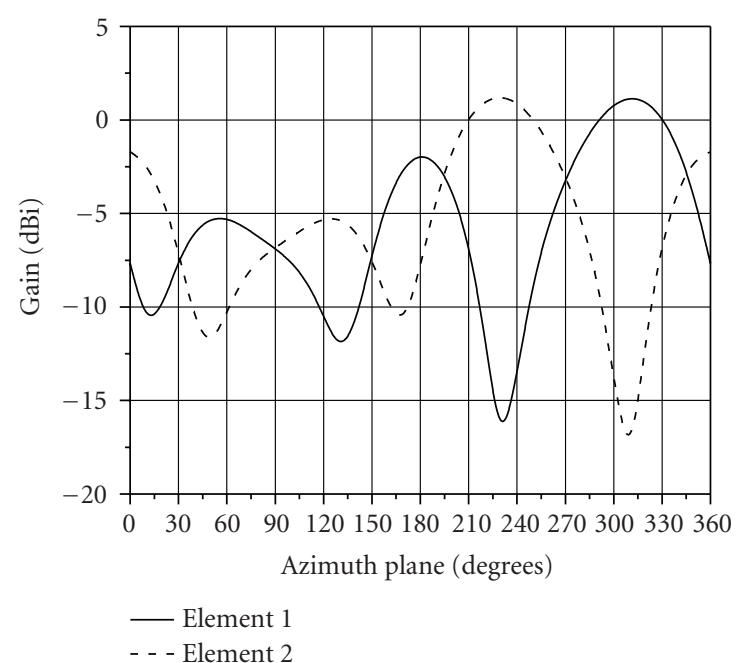

(a)

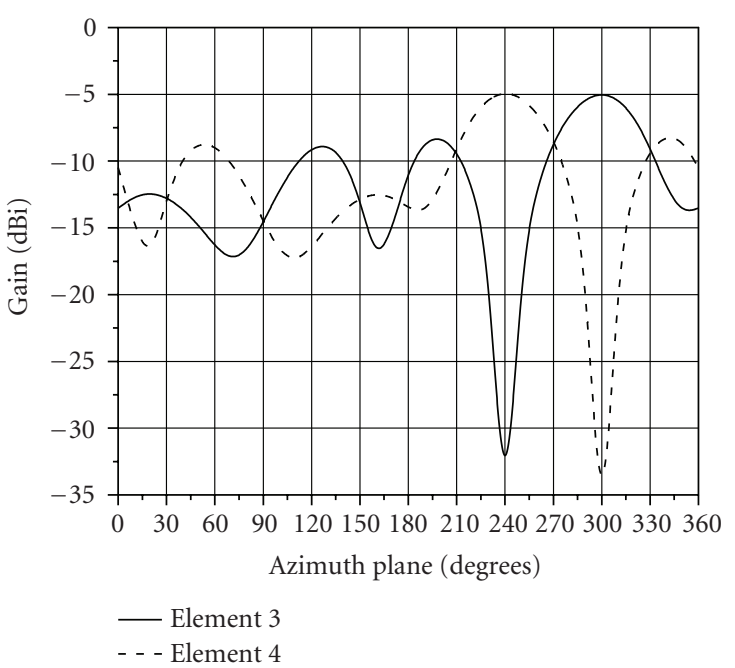

(b)

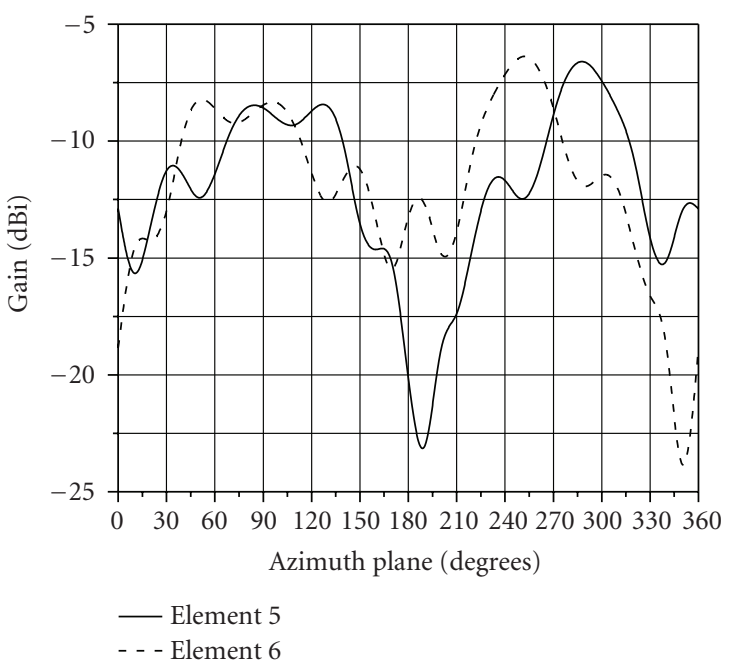

(c)

FIGURE 4: Simulated azimuth plane $(\mathrm{x}-\mathrm{y})$ realized active power gain patterns for the three antenna pairs under the worst case of operation. 


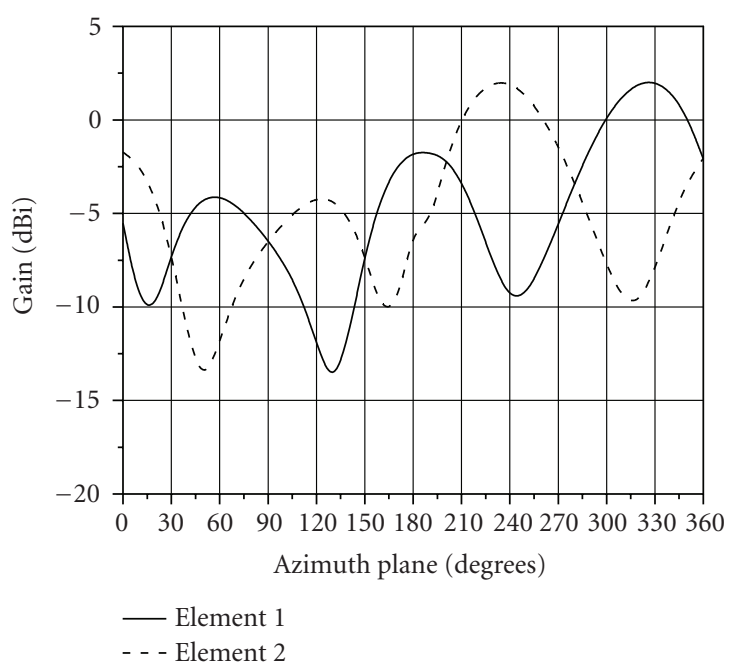

(a)

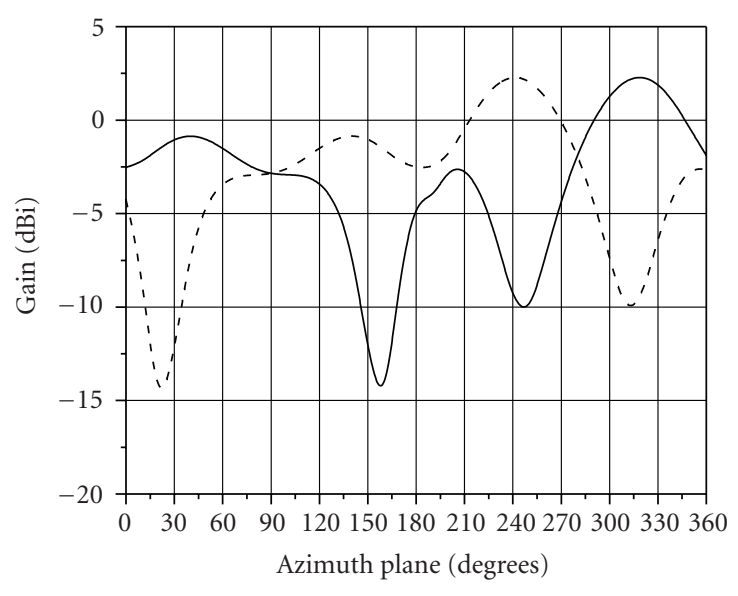

- Element 3

- - - Element 4

(b)

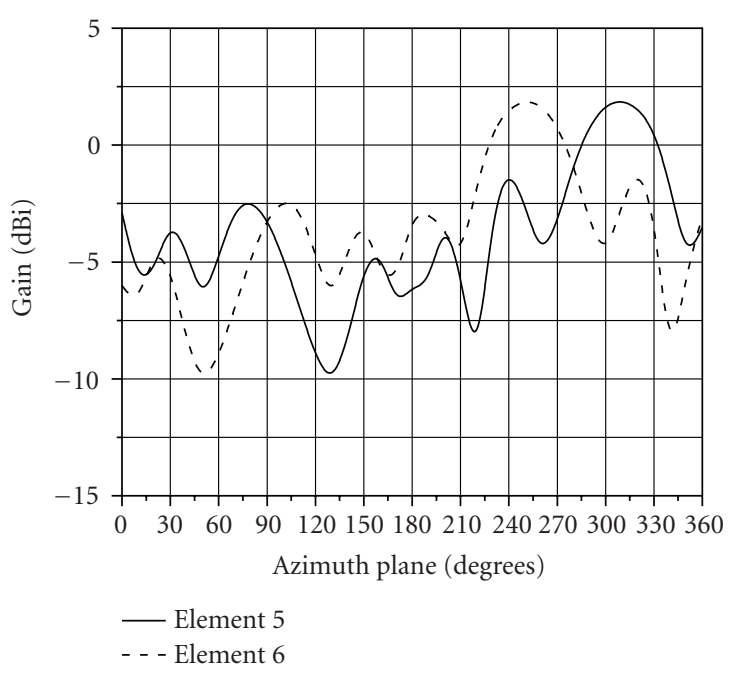

(c)

FIGURE 5: Simulated azimuth plane $(x-y)$ realized active power gain patterns for the three antenna pairs under the best case of operation.
TABle 3: Total efficiency, correlation coefficient, mean effective gains, and effective diversity gain of the multiband diversity system for the best case.

\begin{tabular}{lccccc}
\hline Pair & Frequency $(\mathrm{GHz})$ & $e_{\text {tot }}(\%)$ & $\rho_{e}$ & MEG & EDG $(\mathrm{dB})$ \\
\hline A & 1.8 & 61 & 0.011 & -5.2 & 6.4 \\
B & 2.4 & 75 & $9.6 \times 10^{-3}$ & -4.3 & 7.2 \\
C & 5.2 & 78 & $2.9 \times 10^{-3}$ & -4.1 & 7.5 \\
\hline
\end{tabular}

rest are retained, open-circuited. The realized active power gain patterns for the best case of operation differ from those of the worst case due to the fact that different termination has been used. These antenna patterns on the azimuth $(x-y)$ plane are shown in Figure 5, where the complementary performance due to symmetry is again obvious.

For this operation mode, the mean value of the gain patterns is higher than before, which is caused by the absence of the interband coupling. Table 3 lists the total efficiencies, the envelope correlation coefficients, and the mean effective gains of the antennas for the best case. The total efficiencies and thus MEGs, according to (3), are also improved since no extra power is consumed in the elements of the nonactive antenna pairs. In every case, the calculated values of $\rho_{e}$ are far below 0.5 as in the previous mode.

Regarding the effective diversity gain, the antenna pair $\mathrm{C}$ exhibits again the best performance with $7.5 \mathrm{~dB}$ of effective diversity gain at $5.2 \mathrm{GHz}$, whereas pairs $\mathrm{A}$ and $\mathrm{B}$ provide 6.4 and $7.2 \mathrm{~dB}$ gains at 1.8 and $2.4 \mathrm{GHz}$, respectively, (see Table 3). The undesired impact of the interband mutual coupling has been removed, causing an improved diversity performance for the three investigated frequencies.

\section{CONCLUSIONS}

In this study, a novel compact multiband diversity antenna system for mobile and WLAN applications has been proposed. The simulated results indicate that the antenna structure is suitable for a variety of wireless technologies in the $1.8 / 2.4 / 5.2 \mathrm{GHz}$ frequency bands. The diversity performance in a uniform multipath environment was quantified through the effective diversity gain calculated using the signals covariance matrix for two extreme modes of operation. In any case, the diversity criteria are fulfilled, and the calculated diversity gain offered by the proposed system for the worst/best case of operation is 5.8/6.4, 5.8/7.2, and 7.1/7.5 dB for the 1.8, 2.4, and $5.2 \mathrm{GHz}$ frequencies, respectively.

\section{REFERENCES}

[1] T.-Y. Wu, S.-T. Fang, and K.-L. Wong, "A printed diversity dual-band monopole antenna for WLAN operation in the 2.4and 5.2-GHz bands," Microwave and Optical Technology Letters, vol. 36, no. 6, pp. 436-439, 2003. 
[2] K.-J. Kim and K.-H. Ahn, "The high isolation dual-band inverted $\mathrm{F}$ antenna diversity system with the small $\mathrm{N}$-section resonators on the ground plane," Microwave and Optical Technology Letters, vol. 49, no. 3, pp. 731-734, 2007.

[3] G. Chi, B. Li, and D. Qi, "Dual-band printed diversity antenna for 2.4/5.2-GHz WLAN application," Microwave and Optical Technology Letters, vol. 45, no. 6, pp. 561-563, 2005.

[4] G. A. Mavridis, J. N. Sahalos, and M. T. Chryssomallis, "Spatial diversity two-branch antenna for wireless devices," Electronics Letters, vol. 42, no. 5, pp. 266-268, 2006.

[5] W. He, R. Jin, J. Geng, and B. Gao, "Multiband antenna system with polarization conversion for WLAN applications," Microwave and Optical Technology Letters, vol. 49, no. 7, pp. 1772-1777, 2007.

[6] Y. Ge, K. P. Esselle, and T. S. Bird, "A class of multi-arm monopole antennas for multi-band wireless/mobile applications," in Proceedings of the Workshop on Applications of Radio Science (WARS '06), Leura, NSW, Australia, February 2006.

[7] G. Tsachtsiris, C. Soras, M. Karaboikis, and V. Makios, "A printed folded Koch monopole antenna for wireless devices," Microwave and Optical Technology Letters, vol. 40, no. 5, pp. 374-378, 2004.

[8] G. F. Tsachtsiris, M. Karaboikis, C. Soras, and V. Makios, "Single and multi element printed minkowski monopole antennas for portable terminal devices," Automatica Journal, vol. 45, no. 1-2, pp. 33-39, 2004.

[9] G. Konstantatos, C. Soras, G. Tsachtsiris, M. Karaboikis, and V. Makios, "Finite element modeling of minkowski monopole antennas printed on wireless devices," Electromagnetics, vol. 24, no. 1-2, pp. 81-93, 2004.

[10] ZelSoftware, IE3D, http://www.zeland.com/.

[11] J. L. Volakis, Antenna Engineering Handbook, McGraw-Hill, New York, NY, USA, 4th edition, 2007.

[12] P.-S. Kildal, K. Rosengren, J. Byun, and J. Lee, "Definition of effective diversity gain and how to measure it in a reverberation chamber," Microwave and Optical Technology Letters, vol. 34, no. 1, pp. 56-59, 2002.

[13] G. F. Pedersen and J. B. Andersen, "Handset antennas for mobile communications: integration, diversity, and performance," in Review of Radio Science 1996-1999, pp. 119-138, Wiley-IEEE Press, New York, NY, USA, 1999.

[14] T. Taga, "Analysis for mean effective gain of mobile antennas in land mobile radio environments," IEEE Transactions on Vehicular Technology, vol. 39, no. 2, pp. 117-131, 1990.

[15] D. F. Kelley and W. L. Stutzman, "Array antenna pattern modeling methods that include mutual coupling effects," IEEE Transactions on Antennas and Propagation, vol. 41, no. 12, pp. 1625-1632, 1993.

[16] R. G. Vaughan and J. B. Andersen, "Antenna diversity in mobile communications," IEEE Transactions on Vehicular Technology, vol. 36, no. 4, pp. 149-172, 1987.

[17] W. C. Lee, Mobile Communications Engineering, McGraw-Hill, New York, NY, USA, 2nd edition, 1998.

[18] K. Ogawa and J. Takada, "An analysis of the effective performance of a handset diversity antenna influenced by head, hand, and shoulder effects - a proposal for a diversity antenna gain based on a signal bit-error rate and analytical results for the PDC system," Electronics and Communications in Japan, Part II, vol. 84, no. 6, pp. 10-23, 2001.

[19] V. Papamichael, M. Karaboikis, C. Soras, and V. Makios, "Diversity and MIMO performance evaluation of compact multi element antennas with common phase center," in Proceedings of the 19th International Conference on Applied Electromagnetics and Communications (ICECom '07), pp. 55-58, Dubrovnik, Croatia, September 2007.

[20] M. Karaboikis, V. Papamichael, G. Tsachtsiris, C. Soras, and V. Makios, "Integrating compact printed antennas onto small diversity/MIMO terminals," to appear in IEEE Transactions on Antennas and Propagation. 

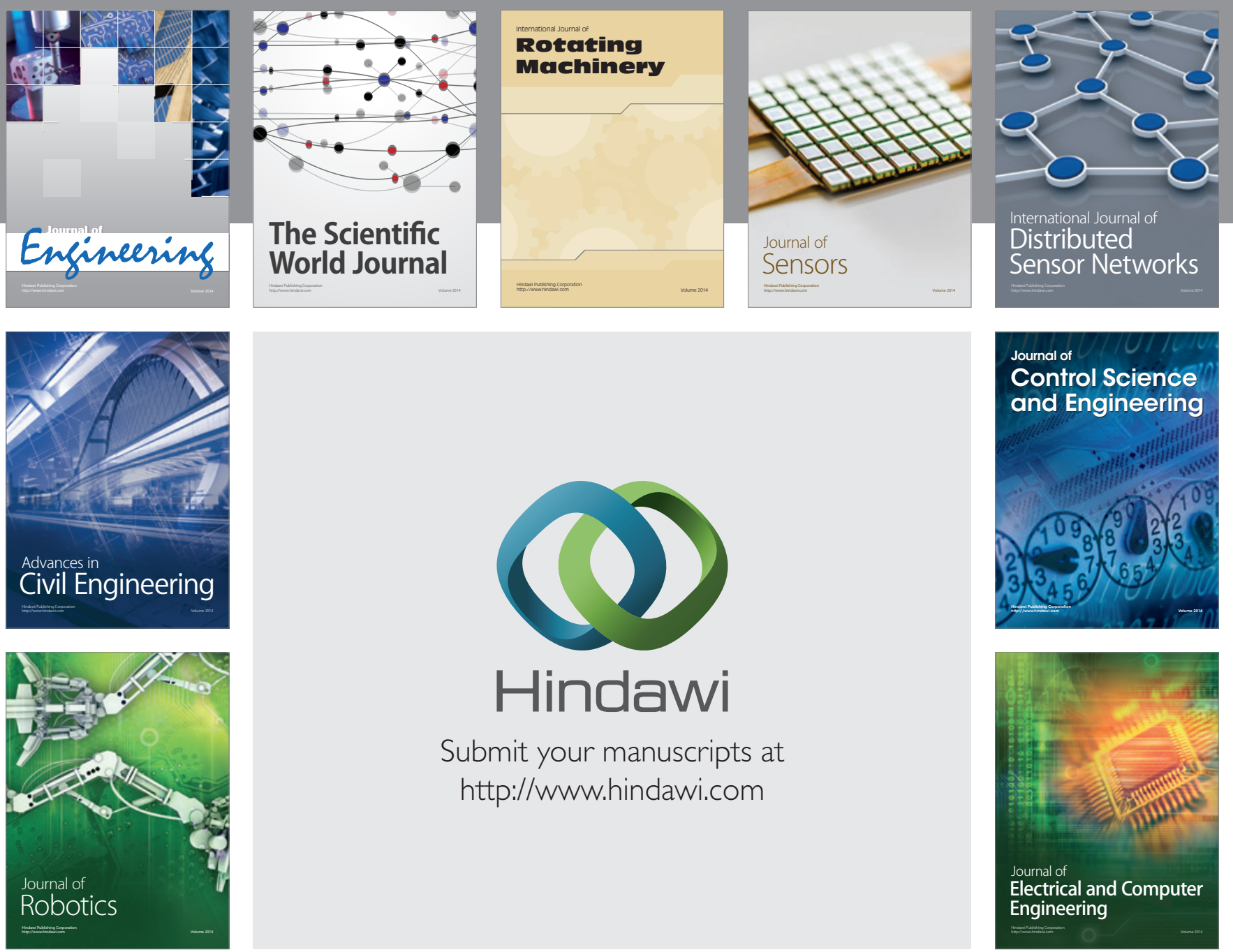

Submit your manuscripts at

http://www.hindawi.com
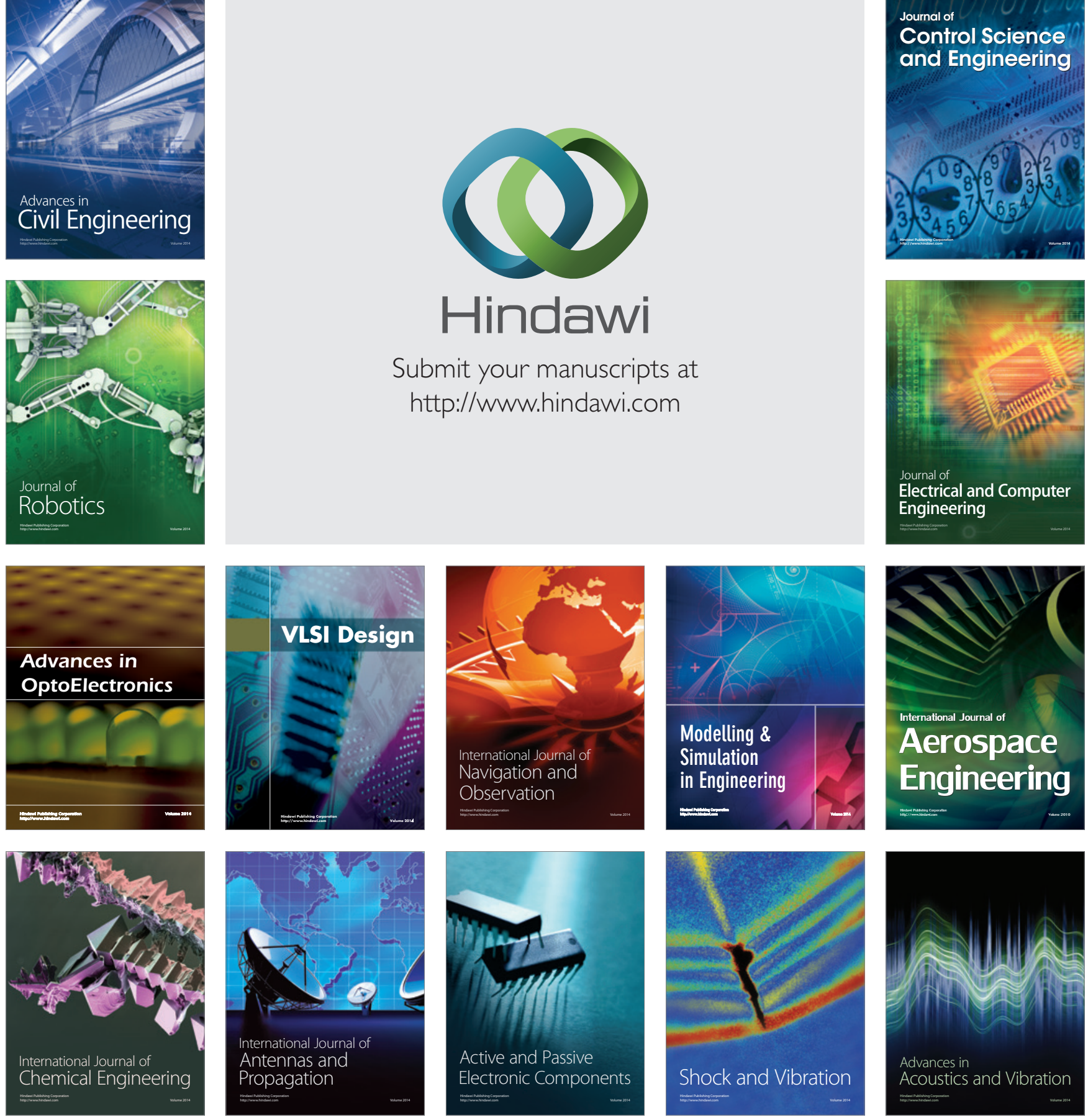